\title{
Das Syndrom der inadäquaten ADH-Sekretion (SIADH)
}

\section{Fallstricke bei der Diagnose und Therapie}

\author{
Andreas Schäffler, Uwe Lindner
}

Die häufigste Ursache für eine euvoläme Hyponatriämie ist das Syndrom der inadäquaten ADHSekretion (SIADH). Auslöser können eine Vielzahl von Erkrankungen und Medikamenten sein. Ist eine kausale Therapie nicht möglich, sind Flüssigkeitsrestriktion, hyperosmolare Kochsalzlösung sowie in Einzelfällen die Gabe von Tolvaptan angezeigt. Dieser Beitrag zeigt anhand von vier Beispiel-Fällen, wie unterschiedlich Ursachen und Erscheinungsbild sein können.

Hyponatriämie - potenziell gefährlich | Die Hyponatriämie (Serum-Natrium $<135 \mathrm{mmol} / 1$ [2]), ist mit einer Prävalenz von etwa 15-30\% [21] die häufigste Elektrolytstörung in der Klinik. Schwere Formen (Werte $<125 \mathrm{mmol} / 1$ kommen in $1-7 \%$ vor $[2,10]$. Die Symptome hängen u.a. davon ab, wie schnell sich die Hyponatriämie entwickelt.

Chronische Hyponatriämien können - auch wenn sie stark ausgeprägt sind - lange Zeit symptomarm verlaufen [7] und dadurch unerkannt bleiben.

Eine unbehandelte Hyponatriämie verlängert den Krankenhausaufenthalt $[5,17]$ und geht mit einer erhöhten Morbidität [20] und Mortalität einher $[2,5,15,18]$. Sturzneigung und Frakturrate sind erhöht $[4,14]$ : In der Geriatrie ist die chronische Hyponatriämie daher von besonderen Bedeutung. Bei Krebspatienten mit Hyponatriämie ist die Überlebensrate signifikant reduziert [13].

Die ungünstige klinische Prognose der Hyponatriämie wird häufig unterschätzt.

SIADH | Abhängig vom Volumenstatus unterscheidet man drei Formen der Hyponatriämie:

- die hypervoläme,

- die hypovoläme und

- die euvoläme Hyponatriämie.

Die euvoläme Hyponatriämie als häufigste Form [7] ist durch einen relativen Wasserüberschuss des Körpers bei normalem Gesamtkörpernatrium gekennzeichnet. Auslöser ist meist ein Syndrom der inadäquaten ADH-Sekretion (SIADH) [2]. Die Bindung von ADH (Vasopressin) an den Vasopressin-2-Rezeptor im Sammelrohr der Niere führt zu einem Einbau von Wasserkanälen (Aquaporin-2) in die Zellmembran. Die Folge: eine Rückresorption von elektrolytfreiem Wasser aus dem Urin [6]. Beim SIADH wird trotz normaler Plasmaosmolalität und arterieller Volumenverhältnisse durch die gesteigerte bzw. inadäquate Sekretion von ADH weniger elektrolytfreies Wasser ausgeschieden, so dass die Natriumkon- zentration im Plasma und damit die Plasmaosmolalität sinken.

Diagnostik | Eine Vielzahl von Erkrankungen können ein SIADH hervorrufen [2, 21]. Auf die Diagnose weisen hin:

- klinische Euvolämie

- erniedrigte Plasma-Osmolalität, inadäquat erhöhte Urin-Osmolalität

- vermehrte Natriumausscheidung im Urin

Für die Urinanalyse reicht Spontanurin aus. Die Parameter sollen möglichst zum gleichen Zeitpunkt bestimmt werden. Wichtig ist, andere Ursachen einer euvolämen Hyponatriämie, v.a. eine manifeste Hypothyreose und eine Nebennierenrindeninsuffizienz auszuschließen ( $\bullet$ Tab. 1 ). Die Serum-ADH-Bestimmung ist nicht erforderlich.

Tolvaptan als Therapieoption I Im Vordergrund steht die Behandlung der Grunderkrankung [9]. Ist dies nicht möglich, kann eine symptomatische Therapie erfolgen:

- Flüssigkeitsrestriktion

- Harnstoff

- hyperosmolare Kochsalzlösung (bei schweren Symptomen) oder

- orale Gabe von Tolvaptan.

Tolvaptan ist ein selektiver Vasopressin-V2-Rezeptor-Antagonist. Er hemmt die ADH-vermittelte Rückresorption von freiem Wasser aus dem Sammelrohr der Niere und führt somit infolge der „Aquarese“ zu einem Anstieg der Serum-Natriumkonzentration [22]. Die Therapie wird unter stationären Bedingungen eingeleitet. Die initiale Dosis beträgt $15 \mathrm{mg} / \mathrm{d}$; sie kann bei Bedarf auf 30 bzw.

Tab. 1 Diagnosekriterien für das SIADH.

\section{Essenzielle Diagnosekriterien}

Hyponatriämie (Serum-Natrium < 135 mmol / I

Plasma-Hypoosmolalität $<275$ mOsm/ kg

Urin-Osmolalität $>100 \mathrm{mOsm} / \mathrm{kg}$

klinische Euvolämie

geteigerte Natriumausscheidung im Urin bei normaler Salz- und Wasseraufnahme

Ausschluss andere Ursachen einer euvolämen Hypoosmolalität 
$60 \mathrm{mg} / \mathrm{d}$ erhöht werden. Die Praxiserfahrung zeigt, dass oftmals niedrigere Dosierungen ausreichend sind. Daher wird eine 7,5 mg-Dosierung aktuell in einer Phase Ib-Studie geprüft.

Überwachung I In den ersten 1-2 Tagen und bis zur Stabilisierung der Tolvaptan-Dosis müssen der Serum-Natriumspiegel und der Volumenstatus mindestens alle 6 Stunden überwacht werden. Die erste Kontrolle des Serum-Natriumspiegels muss spätestens 4-6 Stunden nach Therapiebeginn erfolgen [3].

Cave Bei chronischer Hyponatriämie ( $>48 \mathrm{~h}$ ) darf das Serum-Natrium nicht schneller als $0,5 \mathrm{mmol} / \mathrm{l} / \mathrm{h}$ angehoben werden, da sonst als ernste und potenziell tödliche Komplikation eine zentrale pontine Myelinolyse droht.

Eine Überkorrektur ist ein potenzielles Risiko bei jeder aktiven Hyponatriämie-Behandlung. Eine Flüssigkeitsrestriktion ist bei der Therapie mit Tolvaptan nicht erforderlich; eine zuvor verordnete Flüssigkeitsrestriktion sollte beendet werden. Obwohl Tolvaptan bereits Eingang in die amerikanischen Leitlinien [23] gefunden hat, findet sich eine Empfehlung hierzu in den Europäischen Leitlinien [24] noch nicht.

\section{Fall 1: SIADH unklarer Genese}

Müdigkeit, depressive Verstimmung | Ein 66-jähriger Patient stellt sich in der Ambulanz zur Diabetes-Kontrolle vor. Er leidet seit 10 Jahren an einem Diabetes mellitus Typ 2 ohne Endorganschäden und nimmt Metformin ein, ferner aufgrund einer symptomatischen Hyperurikämie Allopurinol. Der Ehefrau sind Schwäche, Müdigkeit, Konzentrationsschwäche, Interesselosigkeit und depressive Verstimmungen ihres Mannes aufgefallen. Die klinische Untersuchung ist unauffällig. Es werden keine Ödeme und keine Hinweise auf eine Exsikkose festgestellt. Im Routine-Labor fällt eine Hyponatriämie mit einem Serum-Natriumspiegel von $125 \mathrm{mmol} / \mathrm{l}$ auf.
Ursachensuche erfolglos I Eine hyperosmolare Hyponatriämie (entgleister Diabetes) sowie eine Pseudohyponatriämie sind angesichts des Blutzuckers $(111 \mathrm{mg} / \mathrm{dl}[6,16 \mathrm{mmol} / \mathrm{l}])$ und der Triglyzeride $(158 \mathrm{mg} / \mathrm{dl}[1,78 \mathrm{mmol} / \mathrm{l}])$ ausgeschlossen. Auch das $\mathrm{HbA}_{1 \mathrm{c}}$ von 6,3\% belegt die sehr gute Diabetes-Einstellung. Ein ACTH-Test zum Ausschluss einer Nebennierenrinden-Insuffizienz ist ebenfalls unauffällig. Keine Hinweise auf eine paraneoplastische Genese: keine Tumoren der Hypophyse oder des Gehirns, im Magen-Darmbereich, der Prostata und der Schilddrüse [1]. Im Thorax- und Abdomen-CT ist kein Tumor nachweisbar. Allerdings fallen im Thorax-CT zwei $4 \mathrm{~mm}$ große Granulome im rechten Lungenflügel auf ( Abb. 1). Das F-18-D-Glukose-PET zeigt keine pathologische Mehranreicherung, so dass kein eindeutiger Tumornachweis möglich ist. Kein Hinweis auf eine Tuberkulose im QuantiferonTest. Die zusätzlich untersuchten Tumormarker (PSA, NSE, Chromogranin A, Calcitonin, 5-HIES im Sammelurin) sind unauffällig. Auch eine Sarkoidose liegt nicht vor (Bronchoskopie mit BAL, ACE, sIL-2-Rezeptoren).

Symptomatische Therapie I Somit bleibt die Genese des SIADH trotz ausführlicher Differenzialdiagnostik ungeklärt. Nach Einleiten einer Therapie mit Tolvaptan in niedriger Dosierung steigen das Serum-Natrium und die SerumOsmolalität innerhalb von 3-4 Tagen an und die Symptomatik bessert sich deutlich. Zum Ausschluss eines kleinzelliges Lungenkarzinoms wird eine CT-Kontrolle nach 3 Monaten angeordnet. Im Verlauf bleiben die Granulome gleich groß, so dass auch retrospektiv ein Malignom ausgeschlossen ist.

Besserung des kognitiven Zustands I Nach Normalisierung des Serum-Natriums bessert sich der kognitive und psychische Zustand des Patienten deutlich. Auslassversuche führen zu einem Rezidiv - daher erhält der Patient bis auf Weiteres eine niedrig-dosierte medikamentöse Dauertherapie des SIADH. Ein erneuter Auslassversuch ist in 6-12 Monaten geplant.
Abb. $14 \mathrm{~mm}$ großes, größenkonstantes Granulom (roter Pfeil) bei einem Patienten mit SIADH unklarer Genese (high resolution Thorax-CT mit $\mathrm{KM}$, koronar und transversal).
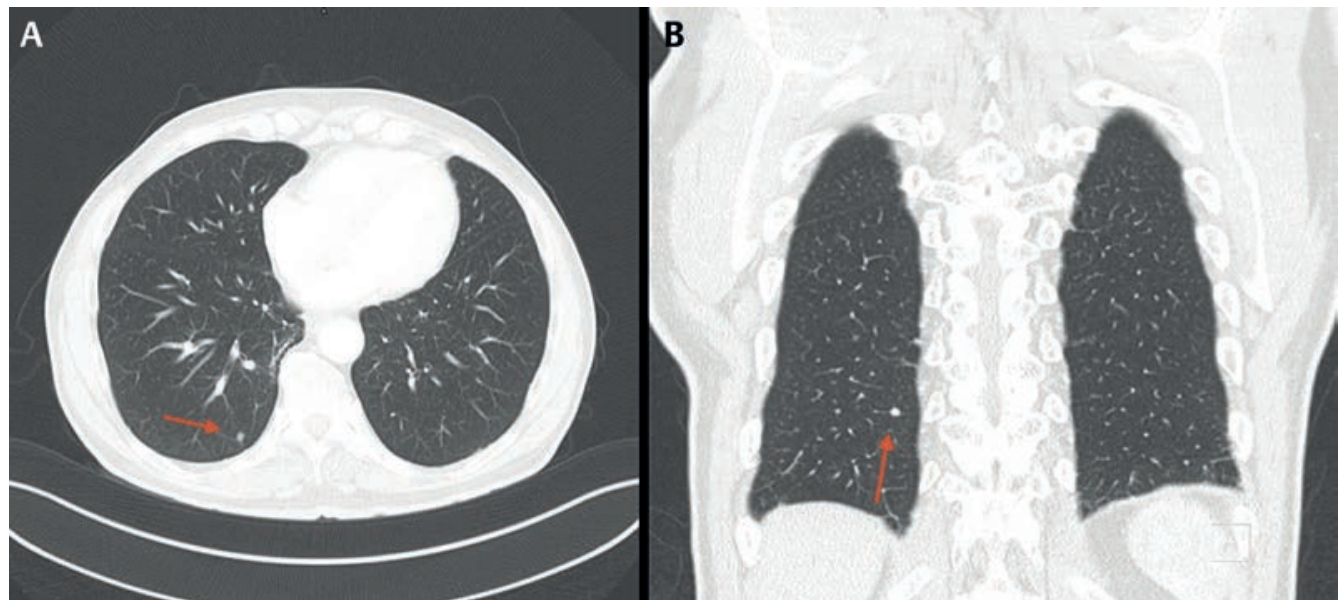
Das SIADH ist häufig eine Zufallsdiagnose. Leichtgradige neurologische Symptome erkennt der Patient selbst oftmals nicht - sie fallen vorwiegend den Angehörigen auf. Da eine Vielzahl verschiedener Erkrankungen zugrunde liegen kann, ist eine sorgfältige differenzialdiagnostische Abklärung essenziell. Dennoch kann die Genese des SIADH nicht immer geklärt werden kann.

\section{Fall 2: SIADH durch Medikamente}

Fallneigung, Desorientiertheit | Ein 75-jähriger Patient kommt aufgrund von Wesensveränderung, Fallneigung, Desorientierung und allgemeiner Schwäche in die Klinik. Infolge eines schweren Schädel-Hirn-Trauma vor 3 Jahren hat er eine schwer einstellbare Epilepsie; seit einem Jahr erhält er Valproinsäure. Bekannt sind eine Linksherzinsuffizienz, KHK, arterielle Hypertonie und ein Diabetes mellitus Typ 2. Der Serum-Natriumspiegel ist mit $119 \mathrm{mmol} / 1$ stark erniedrigt. Isotone Kochsalzlösung bessert die Hyponatriämie nicht.

SIADH durch Valproinsäure I Der Endokrinologe diagnostiziert ein SIADH, mutmaßlich als Folge der Valproinsäure-Behandlung. Eine sekundäre Nebennierenrinden-Insuffizienz oder eine Hypothyreose bestehen nicht. Unter $15 \mathrm{mg} / \mathrm{d}$ Tolvaptan für 2 Tage normalisiert sich das Serum-Natrium; die Symptome bessern sich. Statt Valproinsäure erhält der Patient nun Topiramat; Tolvaptan wird pausiert. Daraufhin bleibt der Serum-Natriumspiegel stabil ( $140 \mathrm{mmol} / \mathrm{l}$ ). Eine erneute Hyponatriämie wird nicht beobachtet.

Medikamente, wie z. B. Valproinsäure, können ein SIADH verursachen. Durch Umstellen auf ein anderes Antiepileptikum kann man eine Normonatriämie und damit eine Besserung der Symptome erreichen. Da die Hyponatriämie ein eigenständiger Risikofaktor für epileptische Anfälle ist, sollte vor der Umstellung jedoch zunächst der SerumNatriumspiegel normalisiert werden.

\section{Fall 3: SIADH nach Gehirn-Operation}

Kopfschmerzen, Schwäche I Eine 57-jährige Patientin kommt aufgrund akut aufgetretener Fallneigung mit Sturz und Synkope, schweren Kopfschmerzen und Schwäche in die Klinik. 9 Tage zuvor war ein Meningeom im Hypophysenbereich (Tuberculum-sellae-Meningeom) operativ entfernt worden ( $\bullet$ Abb. 2 ). Seitdem erhält sie Hydrocortison. Der Serum-Natriumspiegel ist mit $122 \mathrm{mmol} / \mathrm{l}$ im Vergleich zum präoperativen Zustand (145 mmol / l) stark erniedrigt.

Kurzfristige Tolvaptan-Gabe | Die Notfalltherapie erfolgt mit isotoner Kochsalzlösung, unter der sich weder das Serum-Natrium noch die Symptomatik deutlich bessern. Diagnose: akutes postoperativen SIADH aufgrund eines Traumas in der Hypophysenregion. Nach Gabe von Tolvaptan $(15 \mathrm{mg} / \mathrm{d})$ für 2 Tage steigt das Serum-Natrium rasch an (141 mmol / 1); die Symptome bessern sich. Nach Absetzen von Tolvaptan bleiben die Serum-Natriumwerte stabil.

Eine (transiente) Hyponatriämie kann zentralnervös ausgelöst werden, z. B. postoperativ (Trauma in der Hypophysen-Region; sog. Interphase-Problem). Der Hyponatriämie liegt in solchen Fällen in der Regel ein SIADH zugrunde - vorausgesetzt, es besteht keine adrenocorticotrope Insuffizienz. Im Zweifelsfall wird Hydrocortison substituiert, bis die glandotropen Achsen untersucht worden sind. Auch wenn bei komplikationslosen transnasalen, transsphenoidalen Eingriffen kein „cerebral salt wasting syndrome“ (CSWS) beschrieben ist, muss es bei anderen Verletzungen, Blutungen oder Tumoren des Gehirns differenzialdiagnostisch bedacht werden. Gekennzeichnet ist es im Gegensatz zum SIADH durch einen renalen Salz- und Wasserverlust mit Flüssigkeitsdefizit [12].

\section{Fall 4: Hyperkaliämie bei langandauernder Tolvaptan-Einnahme}

Schwäche, Schwindel I Ein 50-jähriger Patient kommt aufgrund von Schwäche, Schwindel und Appetitverlust in die Klinik [16]. 5 Jahre zuvor wurde ein Oropharynxkarzinom (cT3 cN2b G2 M0) und Stimmlippenkarzinom (cT2 G2) mit Cisplatin und Bestrahlung behandelt. Zu dieser Zeit lag der Serum-Natriumspiegel im Normbereich. Ein Tumorrezidiv blieb bislang aus. Im Folgenden sinkt der Serum-Natriumspiegel auf $107 \mathrm{mmol} / \mathrm{l}$. Es wird wiederholt kein Tumorrezidiv festgestellt, jedoch eine COPD (GOLD IV). Medikation: Levetiracetam, Paroxetin, Bromazepam und Lorazepam.

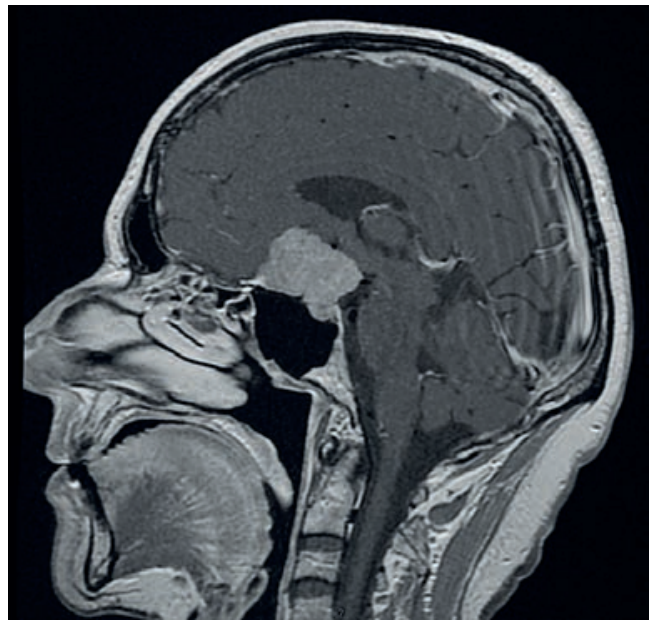

Abb. 2 Tuberculum-sellaeMeningeom. 


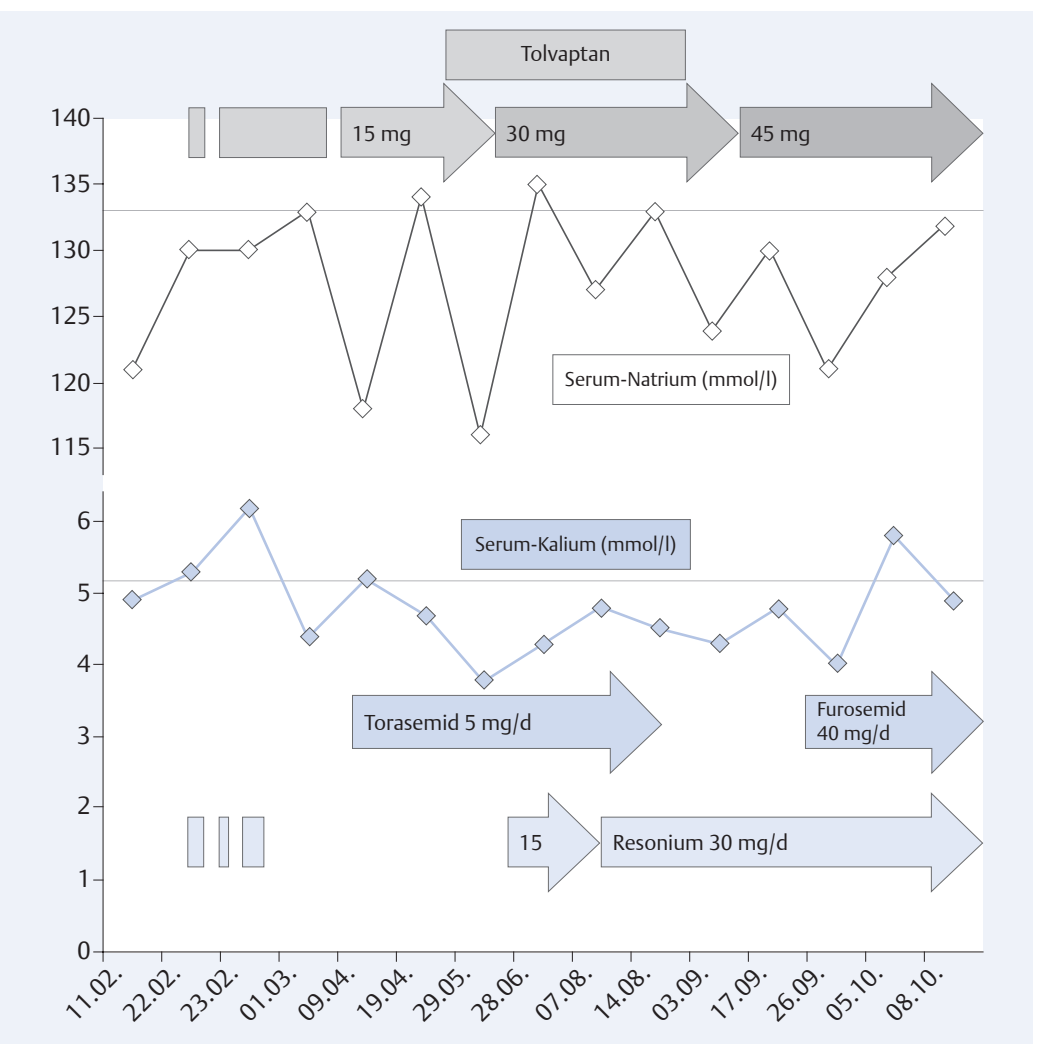

Abb. 3 Serum-Natrium- und Kalium in Relation zur Medikation [16].

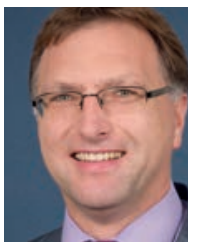

Prof. Dr. med.

Andreas Schäffler

ist Direktor der Medizinischen Klinik und Poliklinik III, Justus-Liebig Universität Gießen, Universitätsklinikum Gießen und Marburg (UKGM), Standort Gießen

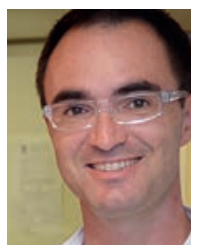

Uwe Lindner

ist Oberarzt und Leiter der Abteilung Endokrinologie an der Klinik für Innere Medizin II, Klinikum Chemnitz gGmbH

DOI 10.1055/s-0041-100836 Dtsch Med Wochenschr 2015; 140: 343-346

(c) Georg Thieme Verlag KG . Stuttgart · New York . ISSN 0012-0472
Differenzialdiagnose des SIADH I Mehrere Ursachen kommen in Frage: das Tumorleiden, die Radiatio, die Antidepressiva oder die COPD. Am wahrscheinlichsten erscheint neben der paraneoplastischen Genese vor allem die Bestrahlung im Halsbereich (Schädigung von N. glossopharyngeus und nervaler Afferenzen des N. vagus).

Schwierige Therapie I Die psychotrope und antiepileptische Medikation werden abgesetzt. Durch Flüssigkeitsrestriktion steigt das Serum-Natrium langsam an und die Symptomatik bessert sich. Aufgrund von schwerer oraler Sicca-Symptome als Folge der Bestrahlung fällt dem Patienten die Flüssigkeitsrestriktion schwer. Er kommt im weiteren Verlauf zweimal mit einer schweren symptomatischen Hyponatriämie ins Krankenhaus. Es wird kein Tumorrezidiv festgestellt, ebenso ergeben sich keine Hinweise auf einen anderen Tumor. Daher wird ein chronisches SIADH aufgrund der Radiatio im anatomischen Verlauf des IX und X. Hirnnervs als am Wahrscheinlichsten angesehen.

Komplikationen I Unter 15 mg / d Tolvaptan steigt das Serum-Natrium rasch und kontrolliert an. Es kommt jedoch zu einer hartnäckigen, chronischrezidivierenden Hyperkaliämie, die mit Natriumpolystyrensulfonat (Resonium $A^{\circledR}$ ) behandelt wird. Kalium-sparende Diuretika oder ACE-Inhibitoren nimmt der Patient nicht ein. Auch endokrinen Erkrankungen mit Hyperkaliämieneigung wie Hypoaldosteronismus oder Hypocortisolismus liegen nicht vor.
Kombinationstherapie | Ein Jahr später reduziert der Hausarzt aufgrund einer erneuten Hyperkaliämie die Tolvaptan-Dosis; er verordnet Torasemid. Daraufhin entwickelt sich erneut eine schwere Hyponatriämie (118 mmol / l) - der Patient wird stationär aufgenommen. Nach Anheben der Tolvaptan-Dosis erhält der Patient kontinuierlich Resonium A eingeleitet. Die Serum-Natrium und -Kaliumspiegel normalisieren sich. Im Folgenden wird der Patient mehrere Male mit einer Hyponatriämie und einer Hyperkaliämie ins Krankenhaus eingeliefert. Die TolvaptanDosis wird von 15 über 30 auf $45 \mathrm{mg} / \mathrm{d}$ titriert. Unter 45 mg/d Tolvaptan, der dauerhaften Gabe von Resonium und einer angepassten Dosierung des Schleifendiuretikums wird schließlich eine Elektrolythomöostase erreicht ( Abb. 3).

Eine Tolvaptan-induzierte Hyperkaliämie kann mit oder ohne renale Vorschädigung entstehen. Sie ist nach Fachinformation „sehr häufig“ und kommt v. a. bei gleichzeitiger Gabe von ACE-Inhibitoren und Kalium-sparenden Diuretika vor. Als Ursache kommt Reduktion des Extrazellulärvolumens durch Tolvaptan in Frage: Gerade bei Vorschädigung der Nieren kann der renale Plasmastrom absinken. Unter VaptanTherapie sind regelmäßige Kaliumkontrollen erforderlich.

\section{Konsequenz für Klinik und Praxis}

- SIADH kann eine Zufallsdiagnose sein. Bei unklaren Laborbefunden ist es ratsam, einen Endokrinologen zur Beurteilung hinzuzuziehen.

- Die Ursachen für ein SIADH sind vielfältig. Häufig lässt sich die zugrundeliegende Genese jedoch nicht (eindeutig) klären.

- Mit Tolvaptan kann die Hyponatriämie schnell, aber kontrolliert normalisiert und dadurch der Krankenhausaufenthalt unter Umständen verkürzt werden.

- Tolvaptan ist sowohl zur kurzzeitigen Behandlung als auch zur Dauertherapie einer Hyponatriämie geeignet.

- Eine sorgfältige Dosistitration, initial unter stationären Bedingungen, ist sehr wichtig.

- Eine Hyperkaliämie ist eine seltene Nebenwirkung, die mit einem lonenaustauscher gut behandelt werden kann.

- Ein Absetzen der Therapie kann schwere Rückfälle zur Folge haben.

Vollständiges Literaturverzeichnis unter http://dx.doi.org/10.1055/s-0041-100836

\section{Interessenkonflikt}

Die aus einem Expertenmeeting resultierende Publikation wurde von Otsuka Pharma (Tolvaptan) finanziell unterstützt. Für die redaktionelle Arbeit sei Dr. med. Monika Vogt und Dr. med. Michaela Ruhmann gedankt. AS und UL haben Vortragshonorare von Otsuka Pharma erhalten. AS und UL nehmen an Advisory Board-Sitzungen bei Otsuka Pharma teil. AS hat an der SAMSCA-PASS-Studie teilgenommen. 
Literatur

1 Beier F, Moleda L, Guralnik V et al. Papillary thyroid cancer associated with syndrome of inappropriate antidiuresis: a case report. J Med Case Rep 2010; 4 110.

2 Ellison DH, Berl T. Clinical practice. The syndrome of inappropriate antidiuresis. N Engl ] Med 2007; 356: 2064-2072

3 Otsuka Pharmaceutical Europe Ltd. Fachinformation Samsca. http://www.zfid.de/pdf/4/012044.pdf

4 Gankam Kengne F, Andres C, Sattar L et al. Mild hyponatremia and risk of fracture in the ambulatory elderly. QJM 2008; 101: 583-538

5 Gill G, Huda B, Boyd A et al. Characteristics and mortality of severe hyponatraemia - a hospital-based study. Clin Endocrinol 2006; 65: 246-249

6 Greenberg A, Verbalis JG. Vasopressin receptor antagonists. Kidney Int 2006; 69: 2124-2130

7 Gross P, Benzing T, Hensen J, Mönig H. Praxisnaher Leitfaden zum Vorgehen bei Hyponatriämie. Dtsch Med Wochenschr 2011; 136: 1728-1732.

8 Hannon MJ, Thompson C]. The syndrome of inappropriate antidiuretic hormone: prevalence, causes and consequences. Eur J Endocrinol 2010; 162: 5-12

9 Hensen J. Behandlung der Hyponatriämie. Internist 2010; 51: 1499-1509

10 Hoorn EJ, Lindemans J, Zietse R. Development of severe hyponatraemia in hospitalized patients: treatmentrelated risk factors and inadequate management. Nephrol Dial Transplant 2006; 21: 70-76

11 Lamas C, del Pozo 2, Villabona C; Neuroendocrinology Group of the SEEN. Clinical guidelines for management of diabetes insipidus and syndrome of inappropriate antidiuretic hormone secretion after pituitary surgery. Endocrinol Nutr 2014; 61: e15-24.

12 Momi J, Tang CM, Abcar AC et al. Hyponatremia-what is cerebral salt wasting? Perm J 2010; 14: 62-65

13 Petereit C, Zaba O, Teber I, Grohé C. Is hyponatremia a prognostic marker of survival for lung cancer? Pneumologie 2011; 65: 565-571

14 Renneboog B, Musch W, Vandemergel X, Manto MU, Decaux G. Mild chronic hyponatremia is associated with falls, unsteadiness, and attention deficits. Am J Med 2006; 119: 71.e1-8

15 Sajadieh A, Binici Z, Mouridsen MR et al. Mild hyponatremia carries a poor prognosis in community subjects. Am J Med 2009; 122: 679-686

16 Schneider A, Schäffler A. A 51-year old patient with $\mathrm{SIADH}$ and hyperkalemia under chronic and high-dose treatment with tolvaptan. Exp Clin Endocrinol Diabetes 2013; 121 - P45.

17 Sherlock M, O'Sullivan E, Agha A et al. The incidence and pathophysiology of hyponatraemia after subarachnoid haemorrhage. Clin Endocrinol 2006; 64: 250-254

18 Stelfox HT, Ahmed SB, Khandwala F et al. The epidemiology of intensive care unit-acquired hyponatraemia and hypernatraemia in medical-surgical intensive care units. Crit Care 2008; 12: R162

19 Suarez JI, Cohen ML, Larkin J et al. Acute intermittent porphyria: clinicopathologic correlation. Report of a case and review of the literature. Neurology 1997; 48: 1678-1683

20 Verbalis JG. Adaptation to acute and chronic hyponatremia: implications for symptomatology, diagnosis, and therapy. Semin Nephrol 1998; 18: 3-19

21 Verbalis JG, Goldsmith SR, Greenberg A et al. Hyponatremia treatment guidelines 2007: expert panel recommendations. Am J Med 2007; 120 (Suppl 1): S1-21

22 Yamamura Y, Nakamura S, Itoh S et al. OPC-41061, a highly potent human vasopressin V2-receptor antagonist: pharmacological profile and aquaretic effect by single and multiple oral dosing in rats. J Pharmacol Exp Ther 1998; 287: 860-867

23 Verbalis JG, Goldsmith SR, Greenberg A et al. Diagnosis, evaluation, and treatment of hyponatremia: expert panel recommendations. Am J Med 2013; 126: S1-S42

24 Spasovski G, Vanholder R, Allolio B et al. Clinical practice guideline on diagnosis and treatment of hyponatremia. European J Endocrinol 2014; 170: G1-G47 